\title{
Short-term Changes in Serum Sex Steroid Levels and Cardiac Function in Healthy Young Men
}

\author{
De Smet $\mathrm{M}^{1}$,2, Lapauw B1 ${ }^{1}$, Kaufman JM11, Ruige JB¹, De Backer T2
}

${ }^{1}$ Department of Endocrinology and 2Department of Cardiology, Ghent University Hospital, Ghent, Belgium.
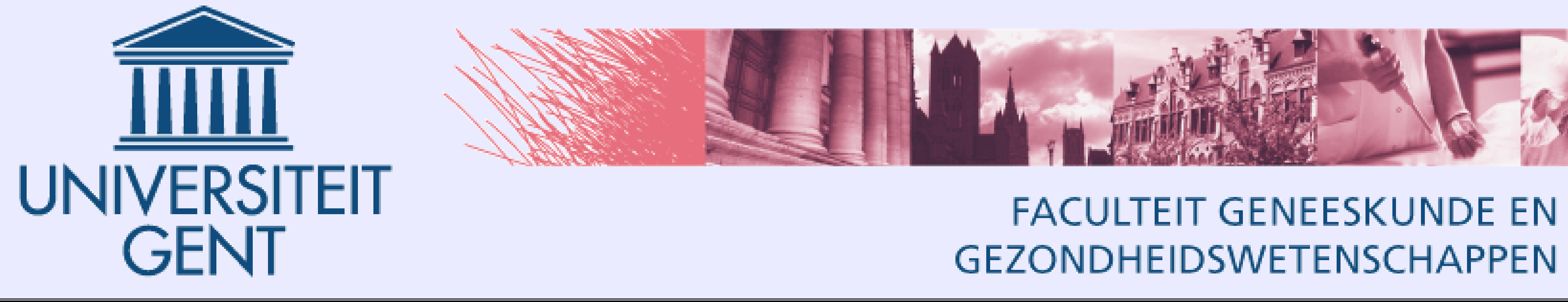

\section{Background \& Aims}

Male obesity is associated with an increase in estradiol $\left(E_{2}\right)$ and a decrease in testosterone (T)

Direct effects of sex hormones on cardiac structure and function are hardly investigated in humans

- To evaluate the effects of contrasting levels of sex hormones as found in obesity on heart function and structure in young and healthy men

\section{Subjects \& Methods}

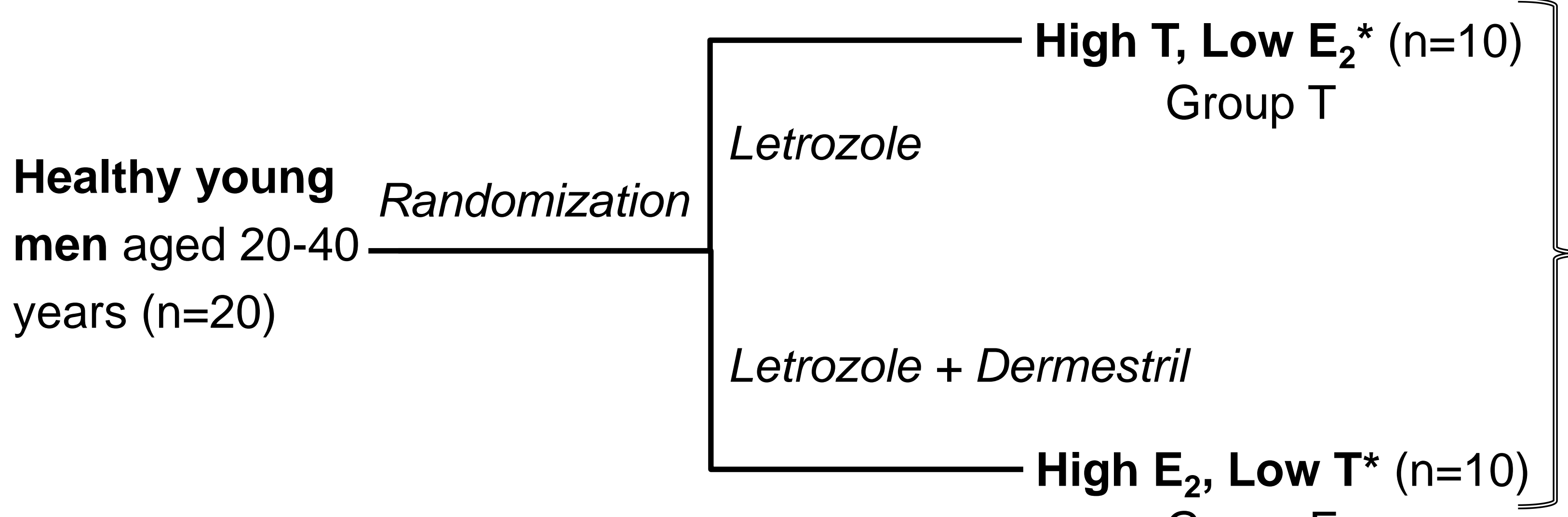

* Serum levels remained within the normal reference range Group $\mathrm{E}$
Basic echocardiography complemented with strain analysis before the intervention and after 7 days

\section{Results}

Total and free $E_{2}$ levels were associated with left ventricular ejection fraction ( $r=0.7, P=0.002$ and $r=0.6, P=0.007$ respectively) at baseline in the whole group $(n=20)$, but not with strain and structural parameters

Total and free T levels were not significantly associated with functional and structural left ventricular parameters at baseline in the whole group

In group E, left ventricular global circumferential strain decreased significantly from $-17.1 \% \pm 3.88$ to $-14 \% \pm 2.46$ after one week as compared to baseline $(\mathrm{P}=0.011)$; however longitudinal and radial strain did not change significantly

No significant changes in cardiac function were observed in group $\mathrm{T}$

Left ventricle diameters, thickness and mass index did not significantly differ before and after the intervention in both groups

\begin{tabular}{|c|c|c|c|c|c|c|}
\hline \multirow[b]{2}{*}{ Parameters } & \multicolumn{3}{|c|}{ Group T (letrozole only) $(n=10)$} & \multicolumn{3}{|c|}{ Group E (letrozole $+E_{2}$ patch) $(n=10)$} \\
\hline & Before & After & $P$ value* & Before & After & $P$ value* \\
\hline Testosterone (ng/dL) & $495 \pm 138$ & $988 \pm 137$ & $<0.001$ & $425 \pm 137$ & $246 \pm 127$ & $<0.001$ \\
\hline Free testosterone (ng/dL) & $8.8 \pm 2$ & $21.5 \pm 4.9$ & $<0.001$ & $9.5 \pm 2.2$ & $5.3 \pm 2.7$ & $<0.001$ \\
\hline Estradiol $(\mathrm{pg} / \mathrm{mL}) \dagger$ & $20.5(17-23)$ & $8.9(8.5-9.4)$ & 0.005 & $16.3(15.1-19.8)$ & $19.4(15.9-41.3)$ & 0.059 \\
\hline Free estradiol (pg/mL) † & $0.37(0.3-0.4)$ & $0.18(0.17-0.2)$ & 0.005 & $0.3(0.28-0.38)$ & $0.36(0.28-0.76)$ & 0.074 \\
\hline Ejection fraction (\%) & $66 \pm 6.16$ & $66.7 \pm 5.7$ & 0.746 & $62.3 \pm 5.17$ & $65.1 \pm 7.61$ & 0.455 \\
\hline Circumferential strain (\%) & $-20.4 \pm 2.88$ & $-20.9 \pm 4.73$ & 0.620 & $-17.1 \pm 3.88$ & $-14 \pm 2.46$ & 0.011 \\
\hline Longitudinal strain, 2-chamber (\%) & $-24.2 \pm 2.58$ & $-23.3 \pm 2.36$ & 0.151 & $-20.9 \pm 2.02$ & $-20.9 \pm 2.34$ & 0.696 \\
\hline Longitudinal strain, 4-chamber (\%) & $-23 \pm 2.68$ & $-23.6 \pm 2.98$ & 0.563 & $-20.9 \pm 3.2$ & $-20 \pm 3.28$ & 0.416 \\
\hline Radial strain (\%) & $48.9 \pm 9.65$ & $50.6 \pm 14.94$ & 0.592 & $52.3 \pm 14.76$ & $47.9 \pm 7.63$ & 0.465 \\
\hline LV mass index $\left(\mathrm{g} / \mathrm{m}^{2}\right) \dagger$ & $85.4(81-102)$ & $84.8(83-94)$ & 0.314 & $99.5(85-112)$ & $99.7(92-114)$ & 0.753 \\
\hline
\end{tabular}

Table 1: Changes in sex steroid profile and cardiac parameters after one week of treatment. Data are means \pm SD or median (1st-3rd quartile) in case of non-Gaussian distribution. ${ }^{*}$ According to paired Student $t$ test. $†$ According to Wilcoxon Signed Ranks test.
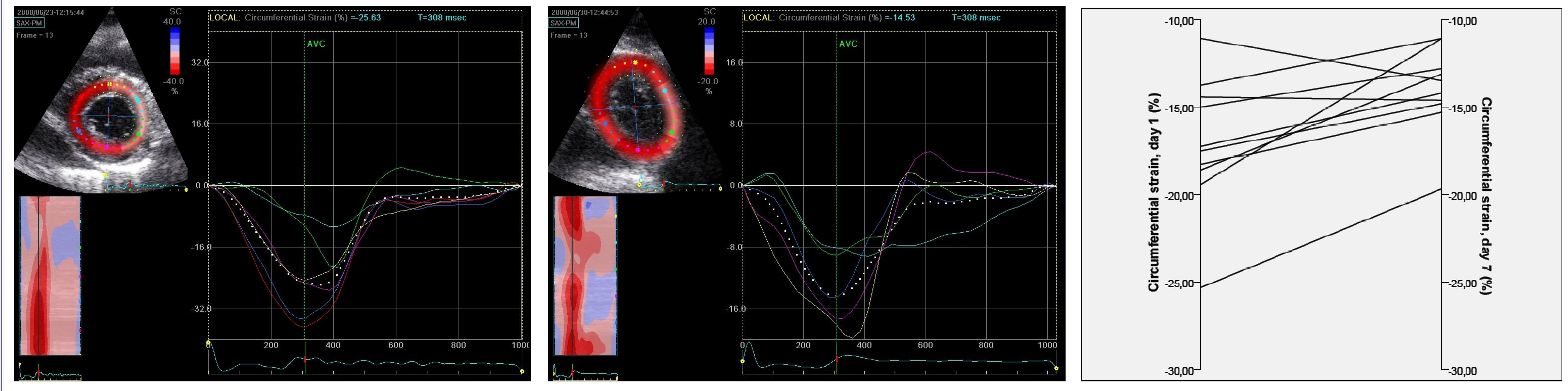

Figure 1: Left ventricular circumferential strain analysis of a patient in group $E$ at the start of the study. The coloured lines represent strain in different regions of the myocardium. The dotted line represents global strair

Figure 2: Left ventricular strain analysis of the same patient after one week. Note the decrease in global circumferential strain. No dyssynchrony observed.
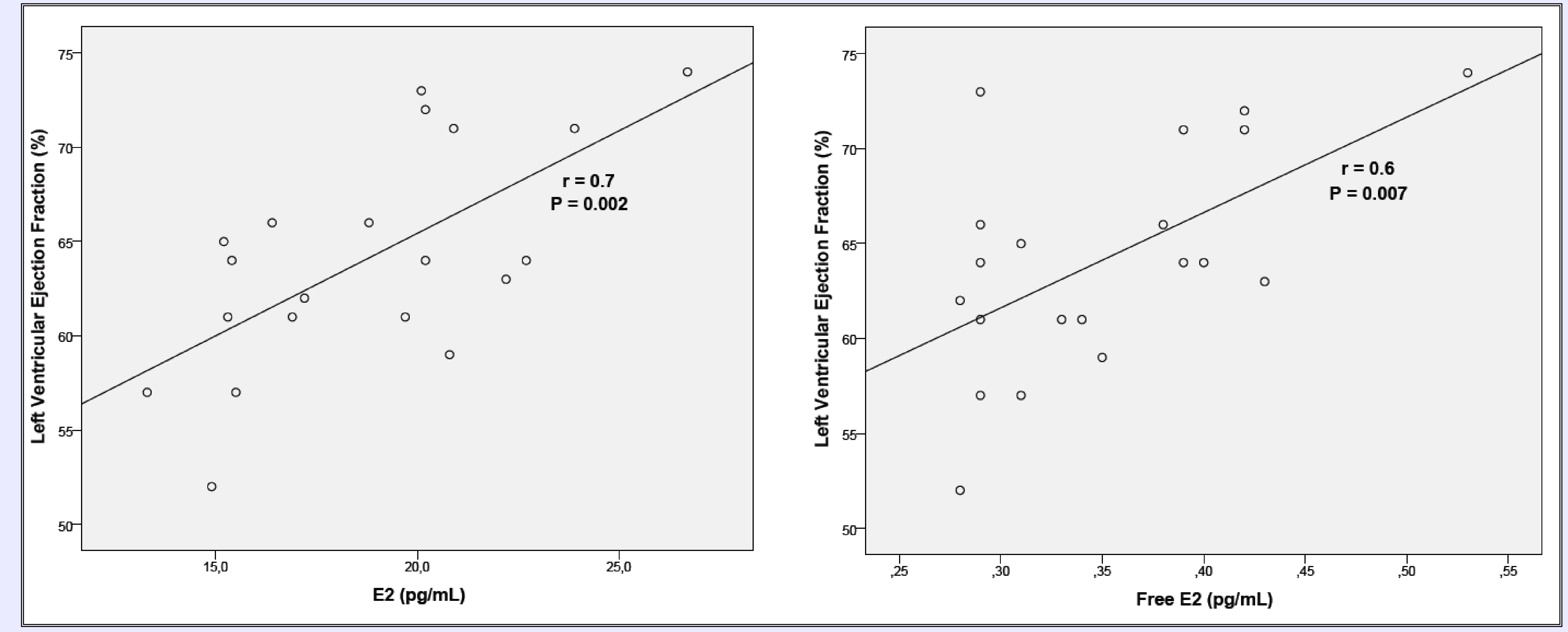

Figure 5: Association between $\mathrm{FE}_{2}$ levels and LVEF

\section{Summary \& Conclusion}

- Short-term administration of letrozole plus $E_{2}$ in healthy young men mimicking changes in sex steroid profile as seen in male obesity, significantly decreased left ventricular global circumferential strain, however longitudinal and radial strain did not change significantly.

At baseline, $E_{2}$ was positively associated with left ventricular ejection fraction, however artificially increasing $E_{2}$ did not improve and may even negatively affect left ventricular function.

The finding justifies larger studies of longer duration to discover the exact nature of the impact of sex steroid profile on cardiac function and remodeling in obesity. 